International Journal of Pure and Applied Mathematics

Volume 94 No. 4 2014, 573-582

ISSN: 1311-8080 (printed version); ISSN: 1314-3395 (on-line version)

url: http://www.ijpam.eu

doi: http://dx.doi.org/10.12732/ijpam.v94i4.13

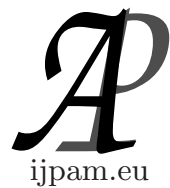

\title{
MATHEMATICAL MODEL OF MAGNETOMETRIC RESISTIVITY SOUNDING FOR CONDUCTIVITY WITH A PSB HOST MEDIUM
}

\author{
P. Haarsa \\ Department of Mathematics \\ Srinakharinwirot University \\ Bangkok, 10110, THAILAND
}

\begin{abstract}
For this paper, we introduce a mathematical model of magnetic response of a two layered earth model which has a constant conductivity in an overburden and a positively skewed bulge conductivity in host medium. The conductivity of ground is a function of depth. Differential and integral equations are applied to for solving a forward problem, and Taylor-series approximation is used to find the solution of the homogeneous differential equation. Numerical solutions are calculated to display the conduct of the magnetic field in graphical forms. The reactions of magnetic field show some points happened as an outcome of the depth and a positively skewed bulge conductivity profile.
\end{abstract}

AMS Subject Classification: 86A25

Key Words: magnetic field, positively skewed bulge, Chave's algorithm, Taylor series, Hankel transforms

\section{Introduction}

The magnetometric resistivity is selected to be one of the most used methods in geophysical exploration. The scheme is established on the estimation of a low level and low frequency magnetic fields connected with a non inductive

Received: May 4, 2014

(c) 2014 Academic Publications, Ltd. url: www.acadpubl.eu 
current flow from the electrodes in the ground. Chen and Oldenburg [3] acquired the magnetic field directly from solving a boundary value problems which was related to the approach used by Edwords [5]. Yooyuanyong and Sripanya [7] obtained and performed the solution of the steady state magnetic field caused a DC current source in three types of heterogeneous earth models. Chaladgarn and Yooyuanyong [8] derived a mathematical model of magnetometric resistivity sounding for a conductive host with a bulge overburden. In this paper, we acquaint a mathematical model and methods for studying the structure of the earth which has a two layered conductive model. We consider the conductivity of overburden is a constant given by $\sigma_{1}(z)=\sigma_{0}, 0 \leq z \leq d$ on the first layer. The conductivity of host medium is $\sigma_{2}(z)=\left(\sigma_{0}+z\right) e^{-b z}, z \geq d$ where $\sigma_{0}$ is a positive constant, $b$ is constant, and $d$ is the thickness of overburden. The conductivity profile in this study is distinct from the models used by Chen and Oldenburg [3] and Chaladgarn and Yooyuanyong [8].

\section{Derivation of Magnetic Field}

The general steady state Maxwell's equation in the frequency domain [3] can be employed to describe the magnetic field for this problem especially

$$
\nabla \times \vec{E}=\overrightarrow{0}
$$

and

$$
\nabla \times \vec{H}=\sigma \vec{E}
$$

where $\vec{E}$ is the vector electric field, $\vec{H}$ is the vector magnetic field, $\sigma$ is the conductivity of the medium in Siemens per meter $(S / m)$ which is supposed to be a function of $z$, and $\nabla$ is the delta operator. By eliminating of $\vec{E}$ from the above equations and leading to get

$$
\nabla \times \frac{1}{\sigma} \nabla \times \vec{H}=\overrightarrow{0}
$$

This can be revealed in a cylindrical coordinates $(r, \phi, z)$ as

$$
\begin{aligned}
& \frac{1}{r}\left[\frac{\partial}{\partial \phi} \frac{1}{r}\left(\frac{1}{\sigma} \frac{\partial r H_{\phi}}{\partial r}-\frac{1}{\sigma} \frac{\partial H_{r}}{\partial \phi}\right)-\frac{\partial}{\partial z}\left(\frac{1}{\sigma} \frac{\partial H_{r}}{\partial z}-\frac{1}{\sigma} \frac{\partial H_{z}}{\partial r}\right)\right] \hat{e}_{r} \\
& +\left[\frac{\partial}{\partial z} \frac{1}{r}\left(\frac{1}{\sigma} \frac{\partial H_{z}}{\partial \phi}-\frac{1}{\sigma} \frac{\partial r H_{\phi}}{\partial z}\right)-\frac{\partial}{\partial r} \frac{1}{r}\left(\frac{1}{\sigma} \frac{\partial r H_{\phi}}{\partial r}-\frac{1}{\sigma} \frac{\partial H_{r}}{\partial \phi}\right)\right] \hat{e}_{\phi}
\end{aligned}
$$




$$
+\frac{1}{r}\left[\frac{\partial}{\partial r}\left(\frac{1}{\sigma} \frac{\partial H_{r}}{\partial z}-\frac{1}{\sigma} \frac{\partial H_{z}}{\partial r}\right)-\frac{\partial}{\partial \phi} \frac{1}{r}\left(\frac{1}{\sigma} \frac{\partial H_{z}}{\partial \phi}-\frac{1}{\sigma} \frac{\partial r H_{\phi}}{\partial z}\right)\right] \hat{e}_{z}=\overrightarrow{0}
$$

where $H_{r}, H_{\phi}, H_{z}$ are the components of $\vec{H}$ in $\hat{e}_{r}, \hat{e}_{\phi}, \hat{e}_{z}$ directions, respectively. Since the problem is axisymmetric, and $\vec{H}$ has only the azimuthal component in cylindrical coordinates. We use $H$ to symbolize the azimuthal component $H_{\phi}$ in the following derivations. By interpreting equation (4), this guides to

$$
\frac{\partial^{2} H}{\partial z^{2}}+\sigma \frac{\partial}{\partial z}\left(\frac{1}{\sigma}\right) \frac{\partial H}{\partial z}+\frac{\partial^{2} H}{\partial r^{2}}+\frac{1}{r} \frac{\partial H}{\partial r}-\frac{1}{r^{2}} H=0 .
$$

where $\sigma$ is a function of the depth $z$ which is provided by $\sigma(z)$. The Hankel transforms [4] is known and defined by

$$
\tilde{H}(\lambda, z)=\int_{0}^{\infty} r H(r, z) J_{1}(\lambda r) d r .
$$

and

$$
H(r, z)=\int_{0}^{\infty} \lambda \tilde{H}(\lambda, z) J_{1}(\lambda r) d \lambda .
$$

where $J_{1}$ is the Bessel function of the first kind of order one, and $\lambda$ is the Hankel variable. Applying the transformation to equation (5), we obtain

$$
\frac{\partial^{2} \tilde{H}}{\partial z^{2}}+\sigma \frac{\partial}{\partial z}\left(\frac{1}{\sigma}\right) \frac{\partial \tilde{H}}{\partial z}-\lambda^{2} \tilde{H}=0
$$

Since the electrode is in the overburden which the end of the electrode is placed at $z=d$ so that a magnetic field will be isolated into two parts. The magnetic field is come from the ground layer that can be explained by the general solutions of equation (8) and the magnetic field arising from probe sources $H$, which is only one element. It can be interpreted by the Ampere's law [7] as

$$
H(r, z)=\frac{I}{2 \pi r} .
$$

where $I$ is the current at the probe on the ground surface. Equation (6) yields

$$
\tilde{H}(\lambda, z)=\int_{0}^{\infty} r\left(\frac{I}{2 \pi r}\right) J_{1}(\lambda r) d r=\frac{I}{2 \pi \lambda} .
$$


By applying the inverse Hankel transforms to the solution of equation (8), we have the magnetic fields in each layer which satisfies the following boundary conditions [1] and [6].

1.The vertical component of the current density must be zero at the ground $\operatorname{surface}(z=0)$,

$$
\left.\sigma_{1}(z) E_{1}^{z}\right|_{z=0}=0
$$

where $E_{1}^{r}$ is the vertical component of the electric field in overburden.

2.The azimuthal component of the magnetic field have to be continuous on each of the boundary planes in the earth,

$$
\lim _{z \rightarrow d^{-}} \tilde{H}_{1}(\lambda, z)=\lim _{z \rightarrow d^{+}} \tilde{H}_{2}(\lambda, z) .
$$

where $H_{1}$ and $H_{2}$ are magnetic field in the first and second layer, in the order.

3.The radial component of the electric field which is given by $E^{r}$ and have to be continuous on each of the boundary planes in the earth,

$$
\lim _{z \rightarrow d^{-}} \widetilde{E}_{1}^{r}(\lambda, z)=\lim _{z \rightarrow d^{+}} \widetilde{E}_{2}^{r}(\lambda, z)
$$

where $E_{1}^{r}$ is the radial component of the electric fields in the first layer, and $E_{2}^{r}$ is the radial component of the electric fields in the second layer. To find out the radial and vertical component of the electric field associated with the azimuthal of the magnetic field, we expand equation (2) and obtain [8]

$$
\begin{aligned}
E= & \left(\frac{1}{r \sigma} \frac{\partial H_{z}}{\partial \phi}-\frac{1}{\sigma} \frac{\partial H_{\phi}}{\partial z}\right) e_{r}+\left(\frac{1}{\sigma} \frac{\partial H_{r}}{\partial z}-\frac{1}{\sigma} \frac{\partial H_{z}}{\partial r}\right) e_{\phi} \\
& +\left(\frac{1}{r \sigma} \frac{\partial}{\partial r}\left(r H_{\phi}\right)-\frac{1}{r \sigma} \frac{\partial H_{r}}{\partial \phi}\right) e_{z}=0
\end{aligned}
$$

Since the problem is axisymmetric and $\vec{H}$ has only the azimuthal component in cylindrical coordinates, we use $H$ to represent the azimuthal component $H_{\phi}$ in the above derivations and yields

$$
E^{r}=-\frac{1}{\sigma} \frac{\partial H}{\partial z}, \quad E^{z}=-\frac{1}{r \sigma} \frac{\partial}{\partial r}(r H) .
$$


Equation (6) can be applied and directed to

$$
\widetilde{E}_{r}(\lambda, z)=\int_{0}^{\infty} r\left[-\frac{1}{\sigma(z)} \frac{\partial}{\partial z} H(r, z)\right] J_{1}(\lambda r) d r=-\frac{1}{\sigma(z)} \frac{\partial}{\partial z} \widetilde{H}(\lambda, z) .
$$

For this geometric model, a two-layered earth model is studied which the interface between the layers is a plane collimate to the ground surface. A point source of direct current $I$ is placed into the overburden for which the tip of the electrode is located at $z=d$. The first layer, the conductivity of overburden is presented by $\sigma_{1}(z)=\sigma_{0}, 0 \leq z \leq d$ on the first layer. The conductivity of host medium is $\sigma_{2}(z)=\left(\sigma_{0}+z\right) e^{-b z}, z \geq d$, where $\sigma_{0}$ is a positive constant, $b$ is constant, and $d$ is the thickness of overburden. By substituting $\sigma_{1}(z)$ and $\sigma_{2}(z)$

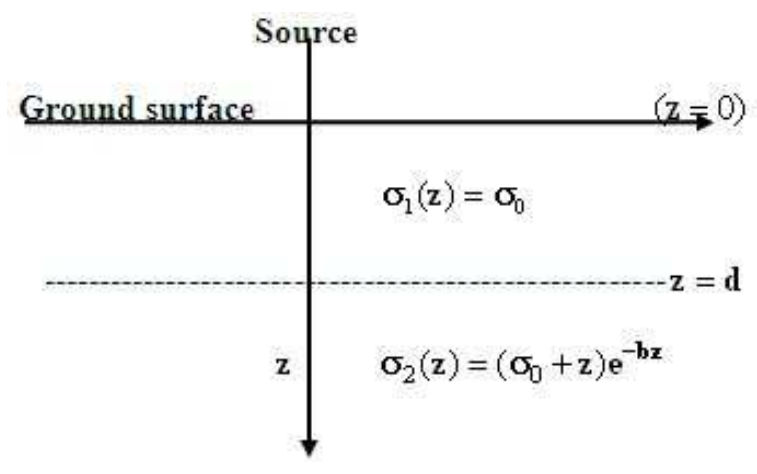

Figure 1: A geometric 2-layered conductive earth model.

into equation (8), we derive the magnetic fields in overburden and conductive host medium as

$$
\frac{\partial^{2} \tilde{H}_{1}}{\partial z^{2}}-\lambda^{2} \tilde{H}_{1}=0
$$

and

$$
\frac{\partial^{2} \tilde{H}_{2}}{\partial z^{2}}-\left(\frac{b z+b \sigma_{0}-1}{z+\sigma_{0}}\right) \frac{\partial \tilde{H}_{2}}{\partial z}-\lambda^{2} \tilde{H}_{2}=0 .
$$

The solution of the homogenous differential equation (16) can be solved by using the Taylor-series approximation, and the auxiliary equation can be applied to find the solution of equation (15). Hence, we have

$$
\tilde{H}_{1}(\lambda, z)=\Psi_{11} e^{-\lambda z}+\Psi_{12} e^{\lambda z}+\frac{I}{2 \pi \lambda} .
$$


and

$$
\tilde{H}_{2}(\lambda, z)=\Upsilon_{11} e^{-\left(\lambda^{2}-\alpha \sigma 0+b\right)(z-d)}+\Upsilon_{12} e^{\left(\lambda^{2}-\alpha \sigma 0+b\right)(z-d)} .
$$

where $\Psi_{11}, \Psi_{12}, \Upsilon_{11}$ and $\Upsilon_{12}$ are arbitrary constants which can be determined by using the boundary conditions. As $z \rightarrow \infty$, we require $\Upsilon_{12}=0$ and equation (18) becomes

$$
\tilde{H}_{2}(\lambda, z)=\Upsilon_{11} e^{-\left(\lambda^{2}-\alpha \sigma_{0}+b\right)(z-d)} .
$$

We apply the first boundary condition into equation (9), we have

$$
\sigma_{1}(z)\left(\frac{1}{r \sigma_{1}(z)} \frac{\partial}{\partial r}\left(r H_{1}(r, z)\right)=0\right) .
$$

For the reason that $\sigma_{1}(z)$ and $r$ are not zero, then

$$
\frac{\partial}{\partial r}\left(r H_{1}(r, z)\right)=0
$$

or

$$
r H_{1}(r, z)=\int_{0}^{\infty} \frac{\partial}{\partial r}\left(r H_{1}(r, z)\right) d r=\int_{0}^{\infty} 0 d r=0
$$

As $r>0$, then $H_{1}(r, z)=0$ and $\widetilde{H}_{1}(r, z)=\int_{0}^{\infty} r H_{1}(r, z) J_{1}(\lambda r) d r$.

Thus,

$$
\Psi_{11} e^{-\lambda z}+\Psi_{12} e^{\lambda z}+\frac{I}{2 \pi \lambda}=\int_{0}^{\infty} r(0) J_{1}(\lambda r) d r=0
$$

Because there are no electric current across at the air-earth interface, that is $I=0$, hence

$$
\Psi_{11} e^{-\lambda z}+\Psi_{12} e^{\lambda z}=0
$$

At $z=0$, we obtain $\Psi_{12}=-\Psi_{11}$. By using the second boundary condition, we derive

$$
\lim _{z \rightarrow d^{-}}\left[\Psi_{11}\left(e^{-\lambda z}-e^{\lambda z}\right)+\frac{I}{2 \pi \lambda}\right]=\lim _{z \rightarrow d^{+}} \Upsilon_{11} e^{-\left(\lambda^{2}-\alpha \sigma_{0}+b\right)(z-d)}
$$


This leads,

$$
\Upsilon_{11}=\Psi_{11}\left(e^{-\lambda z}-e^{\lambda z}\right)+\frac{I}{2 \pi \lambda}
$$

By using of the third second boundary condition, we have

$$
\begin{aligned}
\lim _{z \rightarrow d^{-}} \frac{1}{\sigma_{0}} \frac{\partial}{\partial z}\left[\Psi_{11}\left(e^{-\lambda z}-e^{\lambda z}\right)+\frac{I}{2 \pi \lambda}\right]= & \lim _{z \rightarrow d^{+}} \frac{\lambda}{\left(\sigma_{0}+z\right) e^{-b z}} \\
& \times \frac{\partial}{\partial z}\left[\Upsilon_{11} e^{-\left(\lambda^{2}-\alpha \sigma_{0}+b\right)(z-d)}\right] .
\end{aligned}
$$

Accordingly,

$$
\Psi_{11}=\frac{I \sigma_{0}}{2 \pi \lambda \sigma_{0}\left(e^{-\lambda d}-e^{\lambda d}\right)-\lambda\left(\sigma_{0}+d\right)\left[e^{-d(\lambda+b)}+e^{d(\lambda-b)}\right]} .
$$

and

$$
\Upsilon_{11}=\frac{I}{2 \pi \lambda}\left[1+\frac{\sigma_{0}\left(e^{-\lambda d}-e^{\lambda d}\right)}{\sigma_{0}\left(e^{-\lambda d}-e^{\lambda d}\right)-\lambda\left(\sigma_{0}+d\right)\left[e^{-d(\lambda+b)}+e^{d(\lambda-b)}\right]}\right] .
$$

Hence, by using the inverse Hankel transforms, the magnetic fields in overburden and conductive host medium can be described, in the order, as

$$
H_{1}(r, z)=\int_{0}^{\infty}\left[\frac{I}{2 \pi}-\frac{\sigma_{0}\left(e^{-\lambda z}-e^{\lambda z}\right)}{2 \pi \Omega_{\Delta}}\right] J_{1}(\lambda r) d \lambda .
$$

and

$$
H_{2}(r, z)=\frac{I}{2 \pi} \int_{0}^{\infty}\left[1+\frac{\sigma_{0}\left(e^{-\lambda d}-e^{\lambda d}\right)}{\Omega_{\Delta}}\right] e^{-\left(\lambda^{2}-\alpha \sigma_{0}+b\right)(z-d)} J_{1}(\lambda r) d \lambda .
$$

where $\Omega_{\Delta}=\sigma_{0}\left(e^{-\lambda d}-e^{\lambda d}\right)-\lambda\left(\sigma_{0}+d\right)\left[e^{-d(\lambda+b)}+e^{d(\lambda-b)}\right]$.

\section{Discussion and Conclusion}

For this paper, we show the way to examine the structure of the earth by constructing a mathematical model. We consider a two layered earth model having a constant conductivity profile on the first layer which is given by $\sigma_{1}(z)=$ $\sigma_{0}, 0 \leq z \leq d, \sigma_{0}$ is a positive constant and $d$ is the thickness of overburden. 

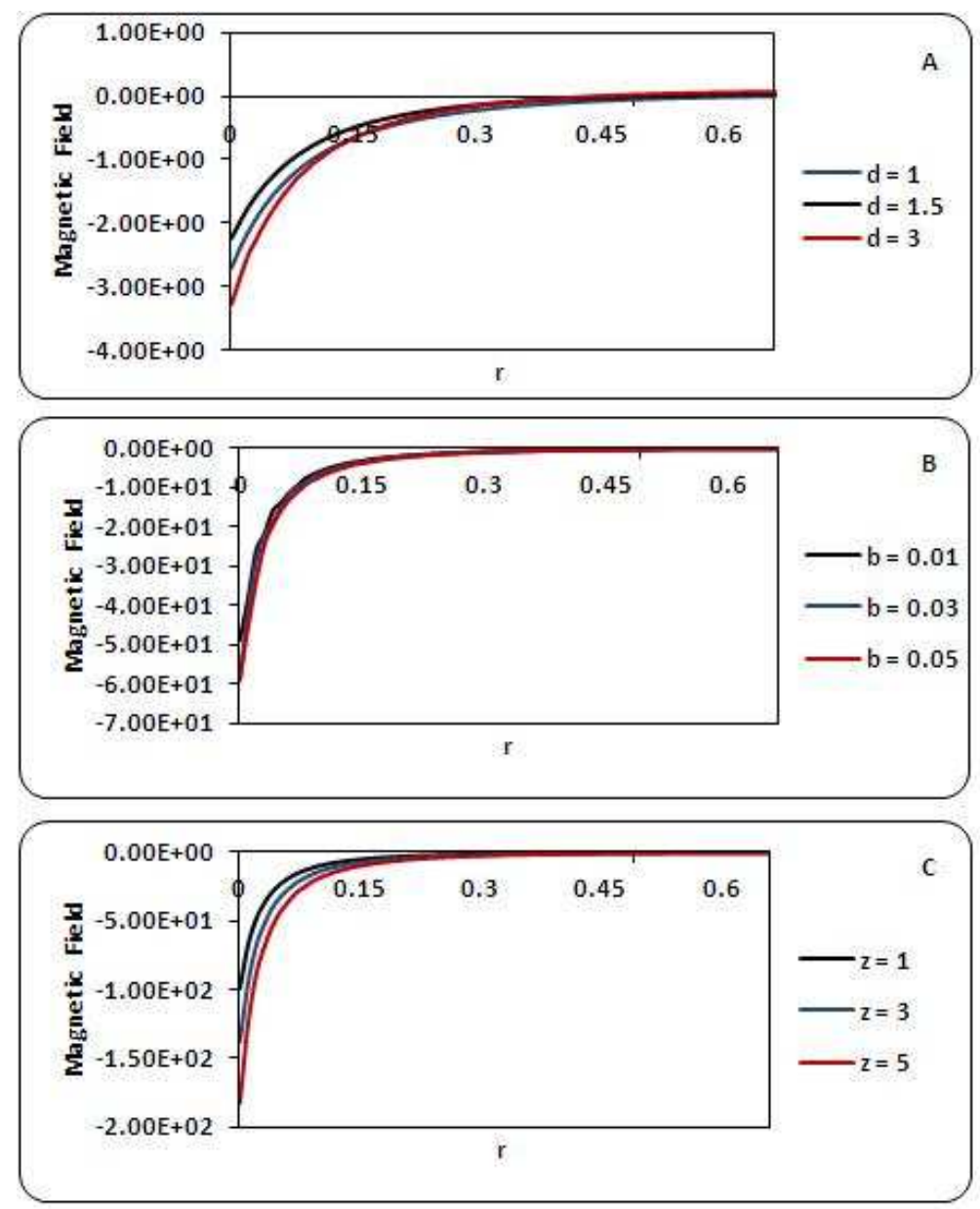

Figure 2: A. Graphs of magnetic field $H$ versus $r$ for a two layered model with a positively skewed bulge conductivity ground profile where $z=1 m, b=0.5 m^{-1}, \sigma_{0}=1 S m^{-1}, d=1 m, d=1.5 m, d=3 m$. B. Graphs of magnetic field $H$ versus $r$ for a two layered model with a positively skewed bulge conductivity ground profile where $z=1 \mathrm{~m}$, $d=5 m, \sigma_{0}=1 S m^{-1}, b=0.01 m^{-1}, b=0.03 m^{-1}, b=0.05 m^{-1}$. C. Graphs of magnetic field $H$ versus $r$ for a two layered model with a positively skewed bulge conductivity ground profile where $d=5 \mathrm{~m}$, $\sigma_{0}=1 S m^{-1}, b=1 m^{-1}, z=1 m, z=3 m, z=5 m$. 
The conductivity of host medium is $\sigma_{2}(z)=\left(\sigma_{0}+z\right) e^{-b z}, z \geq d$, where $\sigma_{0}$ is a positive constant, $b$ is constant on the second layer. We originate the magnetic fields by formulating the problem. The Taylor-series expansion and approximation are used to find the solution of the homogeneous differential equation. Chave's algorithm is performed for numerical calculating the inverse transform of the magnetic field reaction. In our experiments, we force the values of $\sigma_{0}=1 S \mathrm{~m}^{-1}$ and the current $I=1$ ampere throughout the trial while the values of $b, z$, and $d$ are varied. We started off by fixing $z=1 \mathrm{~m}, b=0.5 \mathrm{~m}^{-1}$, $\sigma_{0}=1 \mathrm{~S} \mathrm{~m}^{-1}$ and assigned $d=1 \mathrm{~m}, d=1.5 \mathrm{~m}, d=3 \mathrm{~m}$. We next fixed $z=1 \mathrm{~m}, d=5 \mathrm{~m}, \sigma_{0}=1 S \mathrm{~m}^{-1}$, and assigned $b=0.01 \mathrm{~m}^{-1}, b=0.03 \mathrm{~m}^{-1}$, $b=0.05 \mathrm{~m}^{-1}$. Finally, we fixed $d=5 \mathrm{~m}, \sigma_{0}=1 \mathrm{Sm}^{-1}, b=1 \mathrm{~m}^{-1}$ but assumed $z=1 \mathrm{~m}, z=3 \mathrm{~m}, z=5 \mathrm{~m}$. The magnetic fields were revealed in term of mathematical expressions and plotted as shown in figures $2(\mathrm{~A}, \mathrm{~B}$ and $\mathrm{C})$. The graphs are displayed the behavior of the magnetic field against source-receiver spacing $r$. While the overburden thickness expand, the magnetic fields increase instantly and lead to zero as we anticipated. It is effected not only by the depth $z$ but also by a positively skewed bulge conductivity. We observed that the differences of the graphs shown in figures are counted upon the variation of conductivity.

\section{Acknowledgments}

We would like to thank referees for their valuable comments and suggestions. This work is supported by Faculty of Science, Srinakharinwirot University, Thailand.(grant number 209/2557).

\section{References}

[1] B. Banerjee, B. J. Sengupta and B. P. Pal, Apparent resistivity of a multilayered earth with a layer having exponentiality varying conductivity, Geophys. Prospecting, 28(1980), 435-452.

[2] A.D. Chave, " Numerical integration of related Hankel transforms by quadrature and continued fraction expansion", Geophysics, 48(1983), 1671-1686.

[3] J.Chen and D.W. Oldenburg, Magnetic and electrical fields of direct currents in a layered earth, Expl. Geophys, 77 (2004), 157-163. 
[4] I. Ali and S. Kalla, A generalized Hankel transform and its use for solving certain partial differential equations, J. Austral Math. Soc. Ser B, 41(1999), 105-117.

[5] R.N. Edwords, Downhole magnetometric resistivity technique for electrical sounding beneath a conductive surface layer, Geophysics, 53(1988), 528536.

[6] C.H. Stoyer and J.R. Wait, Resistivity probing of an "exponential" earth with a homogeneous overburden, Geoexploration, 15(1977), 11-18.

[7] S. Yooyuanyong and W. Sripanya, Magnetic field of direct current in heterogeneous ground, Songklanakarin J. Sci. Technol, 29(2007), 565-573.

[8] T. Chaladgarn and S. Yooyuanyong, Mathematical model of magnetometric resistivity sounding for a conductive host with a bulge overburden, Applied Math. Sci., 7(5-8)(2013), 335-348. 